Voigt, C., Unterfrauner, E., \& Stelzer, R. (2017). Diversity in FabLabs: Culture, Role Models and the Gendering of Making. In International Conference on Internet Science. Thesaloniki, GR: Springer.

\title{
Diversity in FabLabs: Culture, Role Models and the Gendering of Making
}

\author{
Christian Voigt ${ }^{1}$, Elisabeth Unterfrauner ${ }^{2}$, Roland Stelzer $^{3}$ \\ ${ }^{1,2}$ Zentrum für Soziale Innovation, Technology and Knowledge, Vienna, Austria \\ voigt@zsi.at, unterfrauner@zsi.at \\ ${ }^{3}$ Happylab GmbH, Vienna, Austria \\ roland.stelzer@happylab.at
}

\begin{abstract}
Diversity and inclusion in the technology sector is increasingly debated, specially in the context of equal opportunities for all and a shortage of experts in many tech related industries. The need to be more inclusive can refer to different age groups, people with diverse culturally and linguistically backgrounds or gender. All in all, ethnic, gender and socio-economic diversity is not yet at the forefront of fabrication laboratories (FabLabs) agendas for change. This paper aims to contribute to the discussion of diversity and inclusion by primarily elaborating gender relations in FabLabs and, to a lesser extent, discussing age and socio-economic conditions of makers. Our analysis is based on 39 interviews and the analysis of 55,450 data points extracted from the log files of 3d-printers, CNC milling machines, laser cutters and cutting plotters. This combination of qualitative and quantitative data reveals that, indeed, some machines are used more frequently by men or women. However, the main difference is in absolute numbers, i.e. women are not joining FabLabs for a variety of reasons ranging from uninviting cultures to the lack of role models in technology driven areas in general.
\end{abstract}

Keywords. Digital Social Innovation · Making $\cdot$ FabLab $\cdot$ Gender $\cdot$ Log File Analysis· Inclusion

\section{Introduction}

"With every new technology, social power relations and thus gender relations are negotiated" [1]. It is a frequent observation that technology has a history of being male dominated and that culture, language and habits are male and often incompatible with femininity [2]. We see socialisation still reproducing gender stereotypes in toys, role models, examples in school books or characters in movies and books. Maker spaces, as a blend of novel technologies but also new practices in developing and producing (almost) everything [3], are confronted with the same male dominance due to their technological nature. The so-called gender gap is often illustrated referencing the substantially lower numbers of female makers to be found in fabrication labs (FabLabs). However, it would be overly simplistic to reduce FabLabs to a set of 
shared machines open to the public. There exist different notions on what making entails, how making differs from hacking or what defines a maker space beyond the machinery found in a physical place [4]. According to [1], FabLabs are places that encourage collaborative learning, knowledge sharing and changing the local economy for the better. The question we want to raise is whether FabLabs can also be places where gender stereotypes are counteracted and to a degree reversed, either by moving technologies into areas such as design, human-computer interaction or generally presenting making as part of a bigger concept around self-expression and selfempowerment. Maker spaces have the potential to provide an experience with technologies that differs from other technology driven areas. We consider these reflections timely because there is a history of failures of initiatives and campaigns pushing for higher participation of women in technology related jobs and activities [5] and this might be a time to push for different or more consistent efforts.

The paper is structured as follows.

- A brief introduction to related works highlights the role of culture, cascading gender gaps and the lack of role models.

- We then discuss data from a recent round of interviews exploring the maker movement as a whole, with a particular focus on gender relations.

- Next, we analyse machine log data from one particular large FabLab and compare the insights from both types of research, the more qualitative interviewing and the more quantitative log file analysis.

- In the conclusion, we argue that the lack of diversity un FabLabs is a societal problem, already starting in schools and families. FabLabs, however, are in a unique position to address this issue and make it part of their agenda for making a difference in how today's society operates.

\section{Related Work: Gendered Artefacts, Gender Gaps and Culture}

That gender relations influence the design of machines is shown in various gender and technology studies, demonstrating that construction, functionality and descriptions of technological artefacts are one-sidedly influenced by male culture [6]. Still, an artefact's gender is not put in stone. A classic example is the microwave, which morphed from a bachelor targeting high-tech gadget into "a female-connoted kitchen appliance" [1].

However, it's not only a desire to get better products when policies aim to increase the share of women in STEM subjects (science, technology, engineering and mathematics). It is also about moving towards a fairer world where women have equal access to high tech jobs and career opportunities. Hence, laying eyes on the numbers only, is potentially neglecting the real challenge, which consists of getting rid of prejudices and misconceptions associated with gender and acknowledge areas where additional action is needed. Equal skills do not automatically lead to equal opportunities, as shown in studies on gaps in pay and promotion [7, 8]. Neither are 'gender gaps' restricted to the tech world, and are also present in early childhood education or nurs- 
ing $[9,10]$, although here a lack of male professionals is noted that receives much less attention than the lack of women in tech.

The maker movement itself already has a strong political agenda that aims to correct a divide between a few producing and many consuming countries. Frugal innovations and the circular economy are concepts trying to empower those with less 'fabrication power' to provide solutions for markets with less 'purchasing power' [11], FabLabs are important creators of opportunities by enabling low cost entrepreneurship [12]. The gender divide, referring to tasks, pay and promotion unduly influenced by gender, could also be addressed by FabLabs, e.g. showing that discriminating genders based on skills is unwarranted. However, for that we need a culture in FabLabs that acknowledges the importance of gender relations without assuming that the 'right culture' will emerge automatically. Liz Henry [13] set up a feminist hackerspace, which at the time was taken as a separatist effort of not wanting to join the larger DIY community. Unfortunately, the debate ignored that the reason for a gender imbalance at maker spaces may be the perceived disrespect or denigration of female makers: "If we aren't at hackerspaces, it isn't because we don't make things, don't code, or aren't technical enough. It's because men act like the space is theirs. Women face harassment ranging from assault to much milder, but more constant, come-ons and innuendos. Our geek cred is constantly challenged or belittled" [13].

\section{$3 \quad$ Research Objectives}

This paper has a twofold objective:

(1) Elaborating the role of gender relations in maker activities. Are there stereotypes or gendered perceptions of the typical female or male maker? Are there different expectations each gender brings to a maker space?

(2) Exploring the different insights from qualitative interviews and quantitative analyses of machine log data. For example, to what degree do insights overlap, complement or contradict each other? Are there activities or use patterns that are dominated by a specific gender?

The first objective was mostly addressed by interviewing makers. A multi-case study of 10 different make spaces [14], ranging from community organised types of maker spaces to maker spaces that were extensions of universities or companies, provided the context for our interviews. The second objective was addressed through the analysis of one of the FabLabs anonymised machine logs, including demographics of members since 2010, machine use statistics since 2012 as well as the type of membership chosen by each member during this time. For example, demographics were available for 3,306 makers in three cities (Vienna, Salzburg, Berlin). Although analytics of activities were later restricted to 2,723 Viennese makers, since they represented the largest share of makers with activity logs going back to 2012. Similarly, a set of six machine groups (3d-printers, CNC milling machines, laser cutter, cutting plotter, PCB etching and a transfer press) produced more than 133,000 data points, of which we analysed 55,450 after selecting the subset for the Viennese Makers. 


\section{$4 \quad$ Interviewing Makers from Diverse Backgrounds}

This chapter shows the analysis of interview data from an ongoing research project ${ }^{1}$ in order to elaborate gender relations in maker activities. The objective here is to see whether the aspects we found in the literature (chapter 2), were also prevalent in the respondents' statements. We start with a brief description of the interviews and the method used for analysing the transcripts. The remaining three sections present interpretations of verbatim quotes, grouped under three themes: culture, gendering and role models.

\subsection{Interviewing and Coding Process}

One of the central aims in qualitative research is to (a) develop hypotheses and support theory building and (b) classify empirical data [15]. Classifying in our context means to systematically order the material following defined classification rules that are informed by theory and are empirically solid in the sense that they have discrimination powers, i.e. an observation can be mapped against an established category without too much ambiguities, even though the operational definition of categories is a known challenge in qualitative research [4]. The interviews were conducted in the context of an EU research project investigating governance, collaboration and value creation in maker spaces [16]. Hence, gender relations were a sub-section of a larger qualitative analysis of these interviews. While there are multiple techniques for interpretation available in qualitative research, classification and structuration were selected as the most appropriate forms for interpreting the cases.

The original material for the analysis is based on the transcripts of 39 interviews, resulting in 576 pages of coded text sections. The coding process was supported through MAXQDA (Figure 1). MAXQDA ${ }^{2}$ is a content analysis tool in qualitative research, supporting coding across multiple files. Codes can be established a priori (deductive) or 'on the fly' (inductive). In this study, we used a deductive coding approach, where the codes are derived from prior hypotheses. The resulting coding tree allows then for easy extraction of test snippets that have been associated with a particular code. The upper part of figure 1, for example, shows the set of documents being analysed and the lower part shows the beginning of the coding system, including sub-codes for main categories such as 'organizing' a makerspace and 'collaborative behaviours' in a makerspace. The final code system had 57 codes in total, but only one code referred explicitly to gender relations.

For the interviews, we chose 10 maker initiatives in 8 countries, following a purposeful sampling approach as described in [17]. The fundamental idea behind purposeful sampling is to make sure that categories of interest - in our case organisation, behaviour and value creation in maker spaces - are considered under as diverse conditions as possible. The exact sampling procedure would go beyond the scope of this paper, but we aimed to maximize variations in four dimensions:

${ }^{1} \mathrm{http} / / /$ make-it.io

${ }^{2} \mathrm{http}: / /$ www.maxqda.com 
- degree of commercial orientation and, if applicable, business model;

- degree of democratization of decision making, e.g. open source principles;

- degree of inclusion of social values in the value propositions;

- scale of interactions at regional, national or global levels.

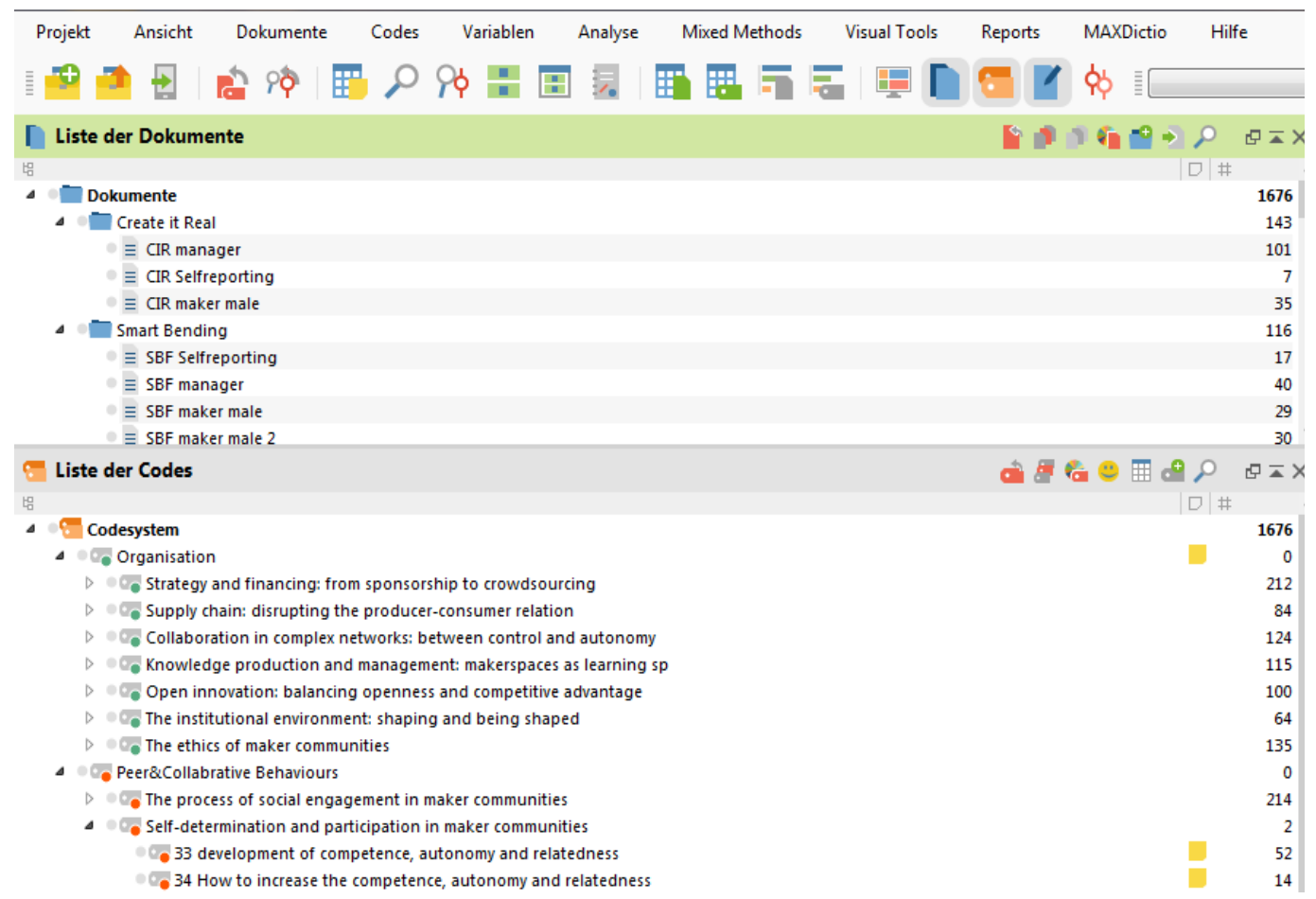

Fig. 1. Content Analysis in MAXQDA (Source: Author's Screenshot)

The resulting maker initiatives included: Happylab Vienna, Austria (HAP); Danish Technology Institute Lab, Denmark (DTI); Fablab Barcelona, Spain (IAAC); Arduino, Italy (ARD); Hochschule Ruhr-West Lab, Germany (HRW); Dezentrale, Germany (Dezentrale); Mini Maker Faire Tartu, Estonia (AHHAA), Fablab Zagreb; Croatia (FLZ); Smart Bending Factory, the Netherlands (SBF); and Create It Real, Denmark (CIR).

The following three sections summarize interview responses related to gender relations. These sections will help to fine-tune future research directions under the headings of culture, gendering and role models.

\subsection{Culture and Values}

Culture is partly defined through what is valued. Or, put more succinctly, maker cultures are characterized by a 'hands-on' imperative, seeking to connect with people and one's environment in ways that allow to shape and change that environment [25]. People's attitude towards their environment is already formed very early on, when youths show different approaches to learning, taking things apart or integrating things [18]. 
In that context, one interviewee emphasized the need to actively promote a culture that appreciates diversity: "And it depends of course also on who you admit and how you deal with mistakes, etc. You can quickly create an environment where making mistakes and also sometimes breaking something is not allowed. And by that you exclude people quickly" (HRW Manager).

Then cultural influences continue in education where a "male-dominated and machine-fixated culture works to marginalize women" [19], directly influencing selfperception. As pointed out by Faulkner [5], the nerdy image of computer hackers and techies is seen as the antipode to the social engagement of women. Women are then forced to make a decision whether to remain within expected gender roles or not: "But in these technical fields I think that boys are not better, but some girls are thinking 'OK, this is not a girl's job' " (FLZ maker). A first step would be, to present gender relations as a serious issue: "[The question of ..] female presence in maker spaces hasn't been noticed much, now it's on our minds. For some time now we have started thinking in these terms - how many girls we have, etc." ${ }^{3}$ (IAAC manager).

Language in general seems to be an important issue, as shown in the following two quotes: "When we speak of the Maker Faire and success stories to the media, I always try to keep in mind that we do not focus on the males only or we risk giving the impression it is an all-male gathering, which is completely false" (AHAA Manager) and "I also noted that a man talking to a woman about technology, he does it in a different way, like he has to explain it more thoroughly - they call it mansplain" (ARD maker). Diversity should not be related to a minority of females in making or hacking. Dedicated events are needed, where female makers and hackers can be among themselves: "Girlsday is always huge at our university, with about 150 female pupils. There, we always try that women or female university students facilitate the courses. That is the special thing about it. Due to the fact that they are among themselves, they are more relaxed" (HRW maker). Similar spaces organised by women for women are Baltazar's Laboratory in Vienna and, in the past, also MzTek in London or Genderchangers in Rotterdam. These places are needed since most maker spaces give influence to those who can spend the most time in a space and "mark territory by gestures and male habitus, resulting in reduced comfort and diversity in a lab" [20]. Not the least it needs firm policies to avoid behavioural aberrations or investigate transgressions of codes of ethics or general norms of conduct: "Well, we don't have posters of pin-up girls in our lockers or things like that and say: 'This is a man's closet and that a woman's.' or similar stories. We are aware of this. At the --- [another maker space] they had a lot of these problems. The atmosphere there made women leave again" (Dezentrale Manager)

${ }^{3}$ Responses were corrected for grammar, however, we refrained from changing interviewees choice of words, hence the use of 'girls' and 'boys' with reference to makers. 


\section{3 'Gendered Task' and 'Gendered Objects'}

Gender-based division of tasks can be commonly observed in today's society. Technologies in modern households are more often used by women than the technologies for home maintenance and gardening, which are predominantly used by men [5].

In some instances, it's just a perception that implies relations between gender and technology: "Women make bio stuff; men make $3 D$ prints ... we also have women coming here now and then to $3 D$ print and laser cut and so on, but the tendency is like that" (Dezentrale manager). Or deterministic thinking about gender is expressed as a reference to past experiences: "Most of the women were in communication, business and design. Now the one we have is doing design but at the same time, she can program" (CIR Manager) or "... [they] already employ female lorry drivers, but generally there are no women wanting to work in these factories" (SBF Manager). Gendered activities are not only imposed from the outside - talking about activities - but is also used in describing, a person's own path of development: "When I was younger I was doing more sewing and simple things for a female. I can't say I have moved to more technical things, but I have learned more topics, and possibly those sewing things are not so interesting anymore. Electronics is more interesting" (AHHAA maker).

Gendered activities were also seen as instrumental in increasing female participation in making: "What is nice about 3d-printing in schools, that you can print jewellery and girls become interested, and some other things for boys" (FLZ manager) or "Laser cutters are typical for girls, because 'My best friend is getting married and I can make a nice cake topper for her' - Pinterest plays a huge part in that ... that's why maker spaces are booming" (HAP, maker). In another case, the instrumentalisation of gendered activities did not work out: "Actually we tried a stitching workshop but it didn't work" (ARD, maker).

Just like activities, objects can be used in a gendered way as well [6]: "in $8^{\text {th }}$ grade some girls printed a unicorn and the boys something like a cover for their mobile phone ... Many also look at what the others do and get inspired by that. If the neighbour says 'I make a mobile phone case', then they make the same thing" (HRW maker). Ideally we can move towards perspectives where activities and objects do not have gender related connotations anymore and are expressed as straight forward problem solving experiences, as shown in this last quote: "I don't remember where I got the idea. At some point I built this greenhouse, with the help of my grandfather, in the countryside. It's not very far away, but we don't go there every day, maybe weekly. It doesn't work well if it's always closed or open, and a couple of years after keeping this greenhouse open all the time, I decided I wanted to make it work properly, and I have the skills to do it" (AHHAA maker).

\subsection{Role Models}

According to Kanter's theory on homo-social reproduction (cloning effects), people are more likely to find their ways into social environments if people with similar interests to their own are already part of the environment [20]. Learning from or working with female role models was also a strong theme in the interviews: 
- The problem that could be, is that if it's a man who is teaching, he would say it in one way, and a girl would say it in another way. They have role models, so if it is a woman saying it, so is it great to look up to her and who she is. It really depends on who is standing there. (CIR maker)

- I do think that by having the Fab Lab attended by a girl is sort of, it makes other women interested in coming to the Fab Lab (DTI Manager)

- For the Girlsday we have looked for female students who implement it, as role models so to say. (HRW maker)

As the few quotes above show, role models are needed in the most diverse situations (teaching maker skills, managing a maker space, organizing an event, etc.). Moreover, what is needed are 'success stories' able to capture the attention of potential makers, and 'success' doesn't need to be interpreted exclusively in terms of business: "There are women, when they are there, who are usually very good and they get noticed quite a lot because they are special. We are setting up a partnership right now with a company where we are taking shares in the company, and the CEO is a woman. ... As you give good examples, as you bring good success stories on women using $3 D$ printers, the end users will evolve" (CIR Manager).

\section{$5 \quad$ Analysis of Machine Log Files}

Whereas the interview material originated from a variety of 10 cases, only the Happylab in Vienna had extensive log files, documenting the use of their machines. In some of the other cases such a system was not available because of a conscious decision to have a self-organizing community without too many regulations. The size of the community plays a role as well. Dunbar's number of 150 describes the size of as group with which we can maintain meaningful, reciprocal relationships, including trusting and relying on each other [21]. The Austrian FabLab whose data we analysed has currently about 2,200 members in three locations: Vienna, operating since November 2010, followed by Salzburg, opening doors in October 2014 and the Berlin facility, which started in September 2016. Hence the mere size of Happylab's membership was a strong motivator to adopt an automated approach to controlling who can access ther machines, based on whether they had the required training or not.

\subsection{Log File Curation}

The log file analysis used data from 5 machine groups (number of data points are given in brackets):

- CNC Milling $(7,004)$

- Laser Cutter $(37,275)$

- Cutting Plotter $(8,281)$

- Printed Circuit Board Etching (526)

- 3D-Printing $(2,364)$

For the first four machine groups actual data on use duration could be analysed, 3d-printing was run through a different system automatically charging makers with 
different material costs. Hence, although we can establish frequency of 3D-printing and number of people doing 3d-printing, no data on the duration of print jobs were available. The comparatively high number of data points for laser cutters is due to the use of a dead man's switch, that required makers to confirm their presence at the machine every 3 minutes. In the first few months of operating the laser cutter, a new data point was created every 3 minutes, which was subsequently changed to only logging start and end times. This rather small detail highlights the importance of scanning even larger datasets for unusual patterns in order to detect and accommodate possible changes of measurement procedures. A further correction of the dataset included dropping point outliers, which can be caused by the faulty reading of a maker's ID card or because the maker didn't log out of the machine at all, until the next person used the machine. In this case we used 98\% winsorization, effectively dropping all data below the 1 st percentile and above the 99th percentile as outliers. Taking the distribution of spending per person as an example, cutting one percent of the data (4 data points) reduces the maximum of money spent from 7,000 $€$ to $2,000 €$, noting that the median of spending is $50 €$ per user in total (independent of his or her membership duration). Nonetheless we are aware that any type of outlier treatment introduces a statistical bias by either under- or overestimating the importance of an outlier. Alternative approaches are outlier classifications based on their median absolute deviation or a-priori thresholds. Hence, by using the percentile-based outlier test we could highlight some extreme values, which we then discussed with the FabLab managers. Figure 2 shows a density plot, where Kernel Density Estimates are calculated via the python package seaborn.distplot [22].

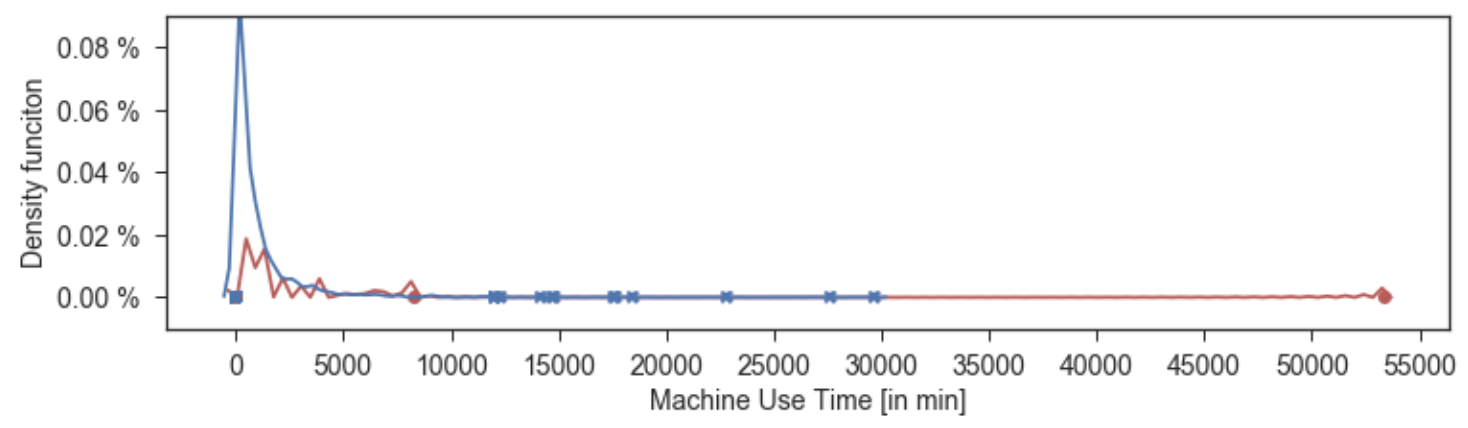

Fig. 2. One per cent outliers for laser cutting (blue crosses) and PCB etching (red dots)

For example, power users could well amount up to 30,000 minutes in total for laser cutting (Median: 437 min, Interquartile Range: 144 - 1,124 min), whereas a value of 53,000 min for the etching printed circuit boards (PCB) seemed highly unlikely (Median: $210 \mathrm{~min}$, Interquartile Range: 105 - $540 \mathrm{~min}$ ).

\subsection{Demographics of Membership}

The following demographic data are restricted to the Viennese location of the FabLab, since maker activities will be discussed primarily in that specific location for the reasons already mentioned. The FabLab's membership is growing steadily until 2015 by more than 200 members each year. Having about 153 members in 2010, the FabLab 
had grown to more than 1627 members in 2016. Growth between 2015 and 2016 had flattened to 46 additional members. Of course, the objective for a physical location cannot be to continuously grow its membership since this could seriously affect the availability of machinery and induce longer waiting times for makers wanting to use a specific machine.

Looking at the percentages of female makers we can also see a steady growth until 2015 , although at a lower rate than male makers. Since 2013 the percentage of female makers remains stable at around 26\% (Figure 3).
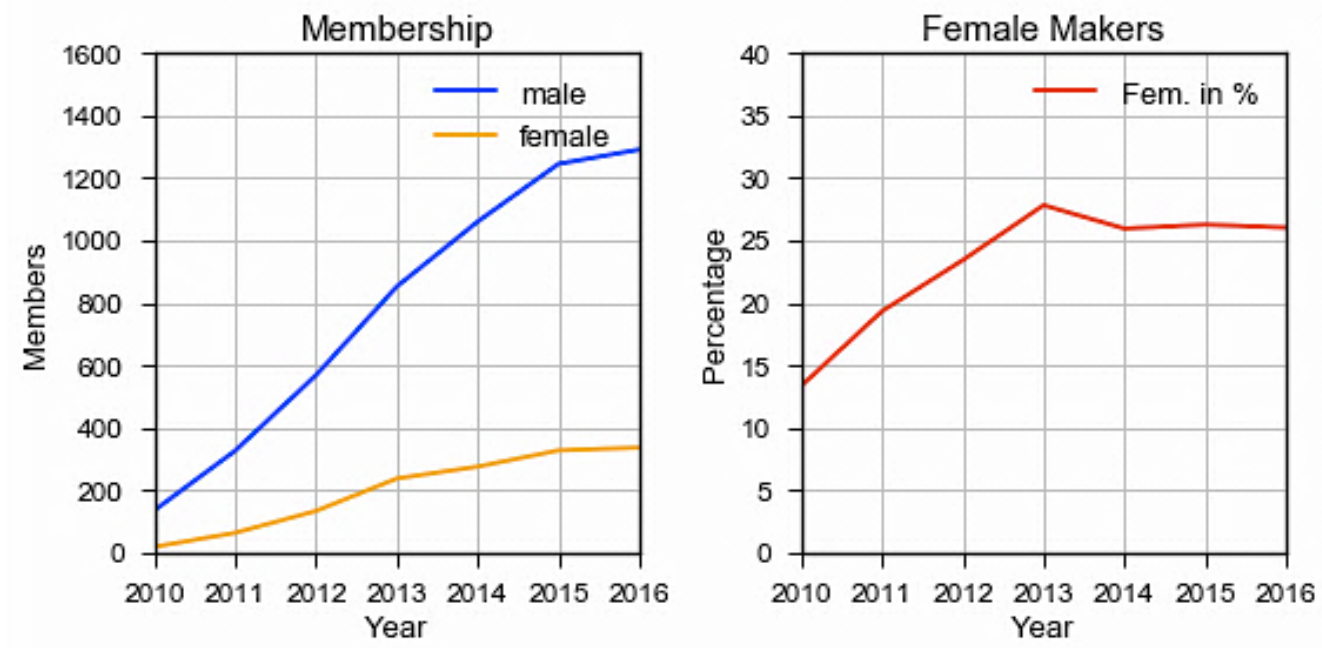

Fig. 3. Membership growth in absolute numbers and proportion of female makers [in \%]
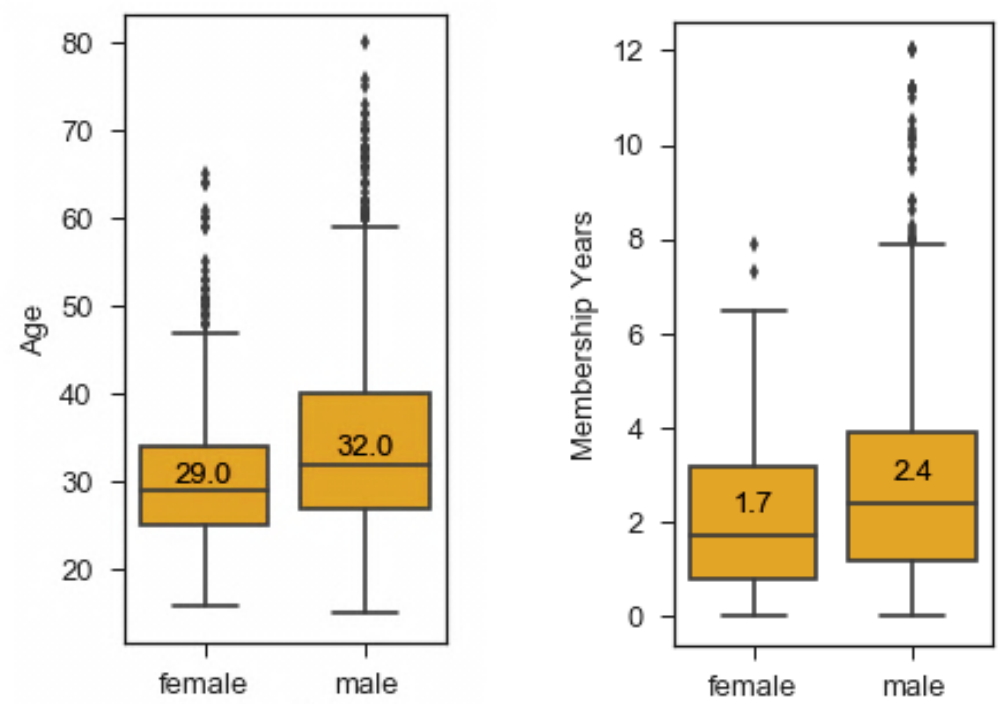

Fig. 4. Median age and membership duration $(n=2714)$

Looking at the age distribution of makers, we can see that male makers are slightly older ( 29 versus 32 years) and that female makers stay shorter with the FabLab (1.7 versus 2.4 years) (Figure 4 ). 
Whereas the age difference might be negligible, the relatively small difference in median membership durations of 0.7 years could actually make a practical difference. Hypothetically, longer memberships in a FabLab could favour more diverse projects and more in-depth knowledge of what the machines can or cannot accomplish. However, further research is needed in order to make more conclusive statements about the influence of membership durations.

\subsection{The Main Benefit of a Larger Membership is Flexibility}

Beside the time someone is a member of a FabLab, another factor influencing his or her ability to actually get to know and use the machines is the type of membership a maker holds. Hence, we were interested in whether male and female makers show differences in their choices of membership types or in the extent to which they use the time available through their membership.

Concerning the Viennese FabLab, there are three types of memberships: (1) small including three half days of four hours, (2) medium - like a small membership plus one extra day and (3) large - 24/7 access to the FabLab. Only looking at the makers who already had a track record in our machine log files, that is 542 male makers and 88 female makers (or 39\% of the membership by the end of 2016), a snapshot shows that about $45 \%$ of male and $50 \%$ of female makers have chosen the small membership (Figure 5), which is a moderate investment of nine Euros per month. The large membership has a 5.4 times higher price tag. Interestingly, the relatively more expensive 'large' membership is not far behind, with $42 \%$ and $38 \%$ of male and female makers respectively. No remarkable differences between genders can be seen at this point.

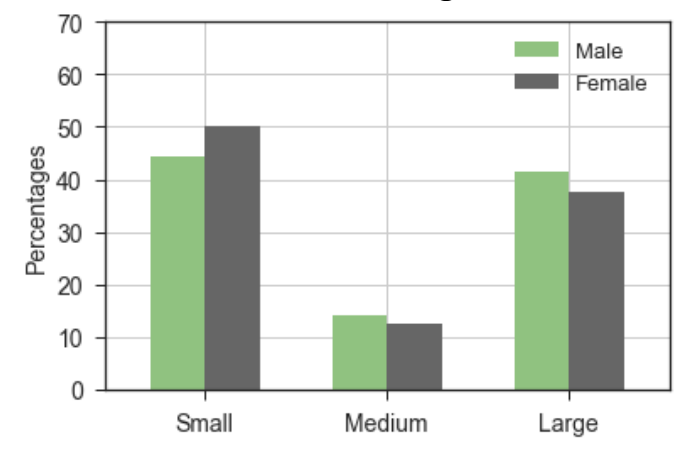

Fig. 5. Memberships in March $2017(\mathrm{n}=630)$

However, the split between the three membership types is constantly in flux, as makers can and do change memberships according to their needs. Looking at the time between 1.1.2012 - 31.3.2017 we analysed membership changes over 275 calendar weeks. Figure 6 shows two membership profiles over time to illustrate the issue. The profile in grey starts in week 30 and has frequent changes between a small and a medium membership and the green profile shows a primarily large membership with two breaks. 


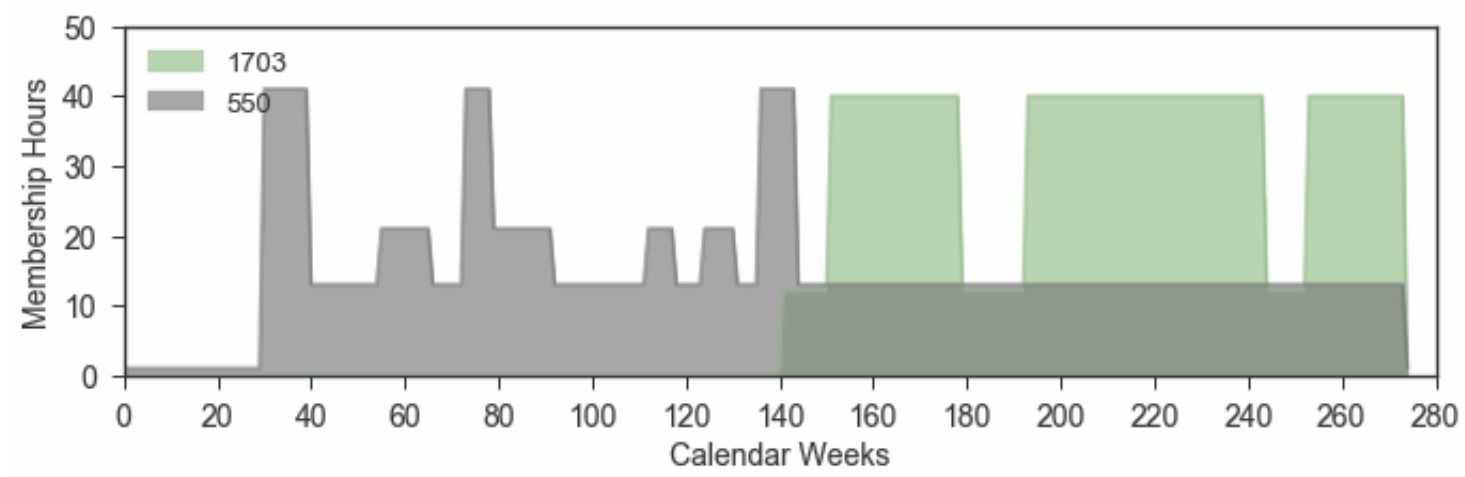

Fig. 6. Membership changes over time

Nonetheless, the number of average weekly hours per person included in memberships remains relatively stable between 19 and 21 hours. Figure 7 shows the development of the three membership types. Approximately every 52 weeks we see a dent in the graph, which reflects the terms and conditions of memberships allowing cancelation only at the end of the year (a condition now removed). Medium and large memberships could be cancelled or upgraded at any time.

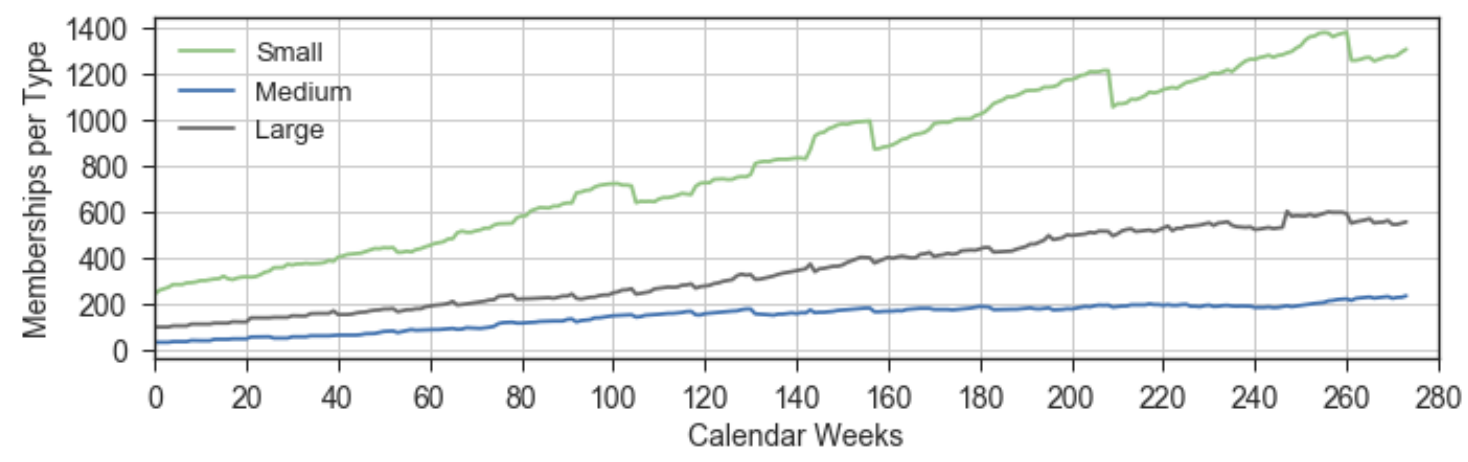

Fig. 7. Development of membership types (including all three locations)

We imagined that a large membership would also be associated with higher aggregated use times of machines. However, as shown in Figure 8 (left side), there is no discernible pattern in the scatter plot, i.e. high use times can be found independently of the time included in a membership.
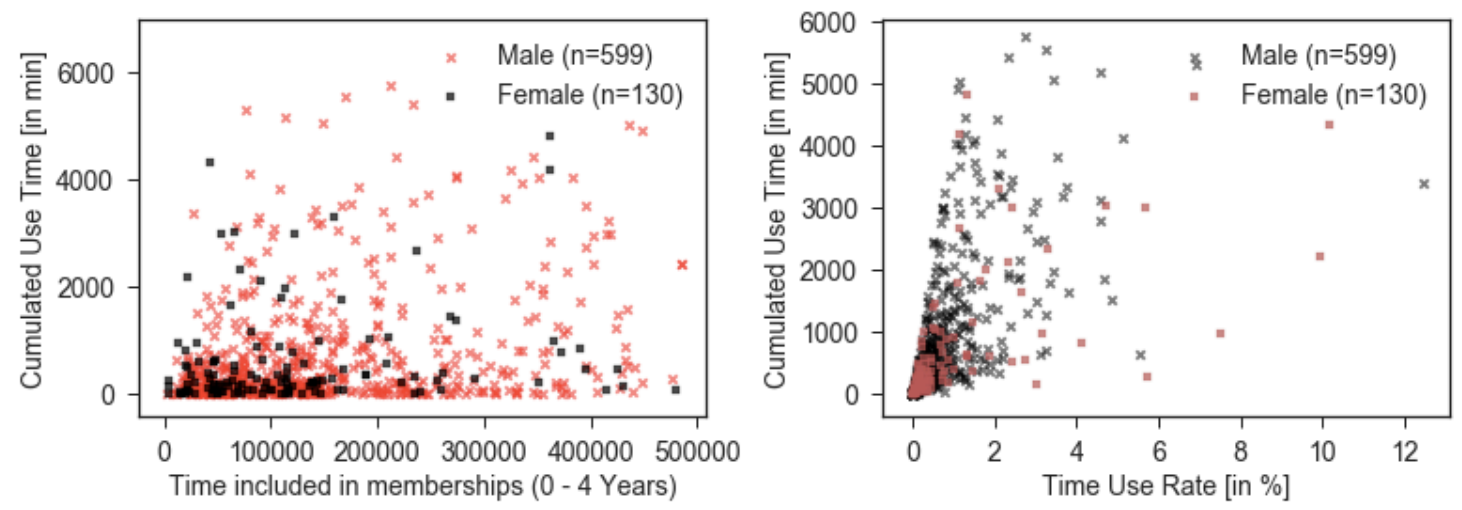

Fig. 8. An approximation to a time use rate 
The right side of Figure 8 confirms the independence of membership time and use rate, whereas 'use rate' is calculated as membership time in minutes divided by total minutes spent on FabLab machines. So far 'use rate' can only be taken as a calculatory proxy, because we do not have records of makers presence apart from the times they use a machine with access control. This implies that times on the electronics workbench, other manual tools or times for 3d-printing are not included. Nonetheless, keeping in mind these analytical limitations, we can see that the 'use rate' for the majority of makers is below $1 \%$. Separating the genders, however, does not show a significant difference for 'use rates' between female and male makers: Mann-Whitney-U test $(\mathrm{U}=38119, \mathrm{p}=.35)^{4}$.

Given the relatively low use rates and the fact that more time included in memberships does not necessarily lead to more time used, we would suggest that makers opt for large memberships because of the flexibility that comes with 'large'. Hence, the main benefit of having access $24 / 7$ is not the amount of time but the fact that the one hour a maker might need can be chosen at the most convenient time for the maker.
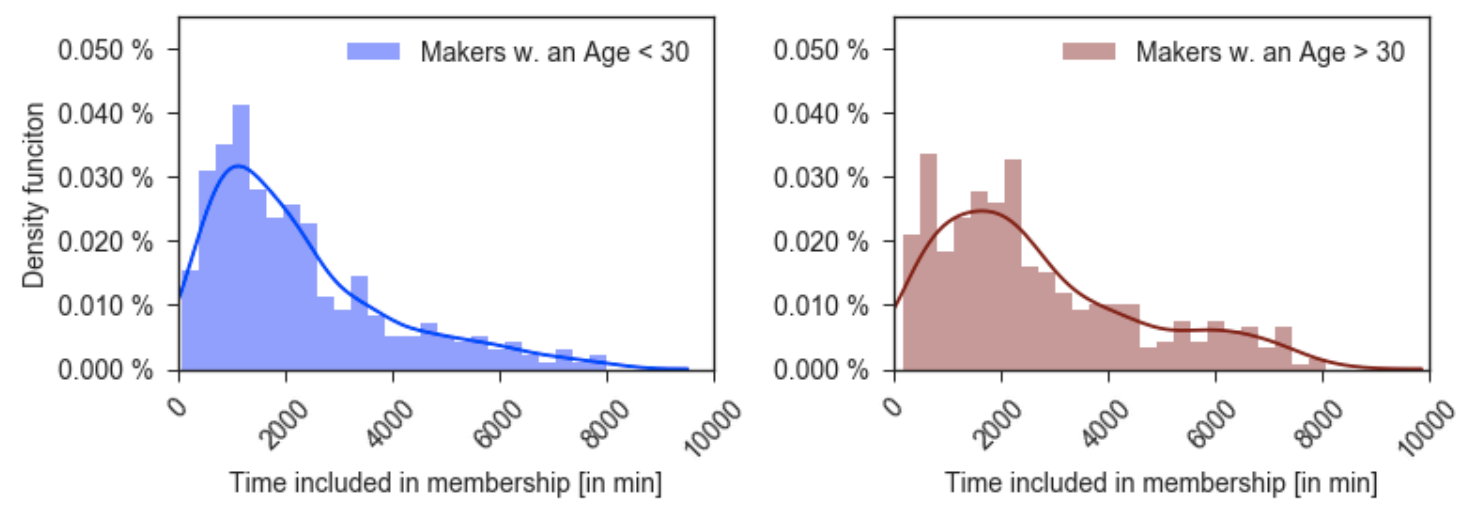

Fig. 9. Time included in memberships for makers below $(n=306)$ and above 30 years $(n=383)$

Of course, this convenience has a price that might be more affordable to makers over 30 years. If we look at two samples of makers, we can see that makers over 30 have more time through their memberships (Median $=2,196 \mathrm{~min}$ ) than maker under 30 years $($ Median $=1,744 \mathrm{~min})$. Testing for group differences, we can say that the probability of a person over 30 years having more hours through their membership is statistically significant: Mann-Whitney-U test $(\mathrm{U}=49122, \mathrm{p}=.0004)$.

\footnotetext{
${ }^{4}$ Since the machine $\log$ data were substantially skewed, i.e. not following a normal distribution, we used non-parametric statistics such as the Mann-Whitney-U test. Non-parametric methods do not require any a priori assumptions about parameters that describe the distribution of data (e.g. a mean or variance). The Mann-Whitney-U test is used when we want to know whether two samples (e.g. a variable split by gender) belong to the same distribution or not. Put differently, the test helps to establish whether there is a statistically significant difference between observations related to male or female makers.
} 


\subsection{Gendered Preferences for Specific Machines}

The next comparison of gender is related to the number of machines a maker uses as well as which machines are used. Of course, which machines are used depends on the maker's interests and projects, however, there is a relationship between knowing the machines and making informed choices about the most adequate way to fabricate a desired object.
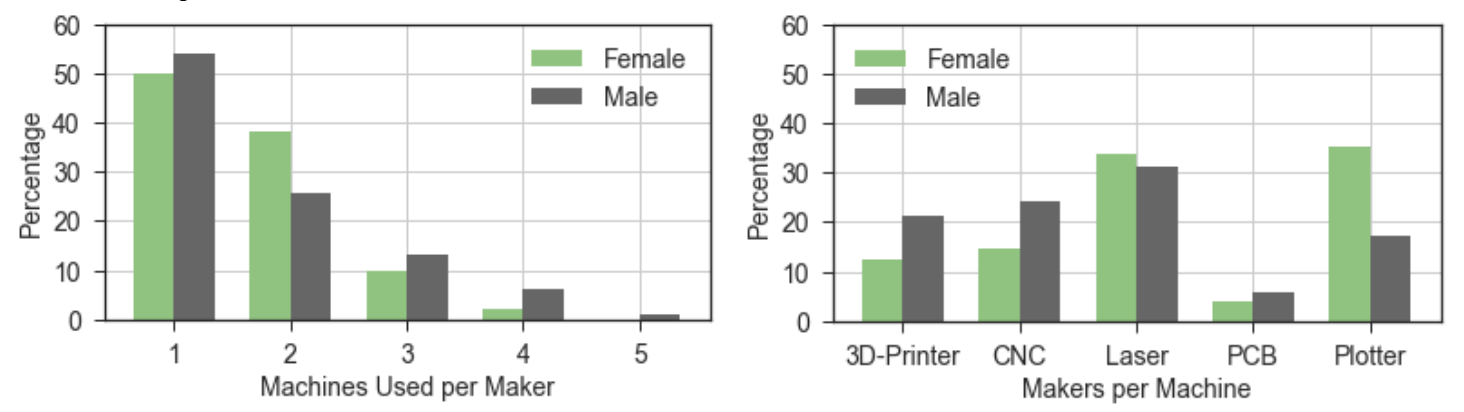

Fig. 10. Multiple machine use and percentages of makers per machine

Given a set of five machines (3d-printer, CNC milling, laser cutter, cutting plotter, PCB etching), $11.8 \%$ of female makers $(n=152)$ use more than two machines compared to $20.3 \%$ of male makers $(n=758)$. In terms of percentages, we can say that $3 d-$ printer and $\mathrm{CNC}$ milling machines are more often used more by male makers, twice as many female makers as male makers use the cutting plotter and laser cutters as well as PCB etching are used to a similar degree by both genders (Figure 10).

In terms of median use time, we see moderate differences between genders: 15 minutes for cutting plotters, 34 minutes for $\mathrm{CNC}$ milling machines and the highest difference of 70 minutes can be found with laser cutters. The median use time for PCB etching was 78 minutes for male makers $(n=88)$. For female makers the overall number for using PCB etching was already quite low $(n=8)$ and restricting it to the first two years, only two female makers were counted.

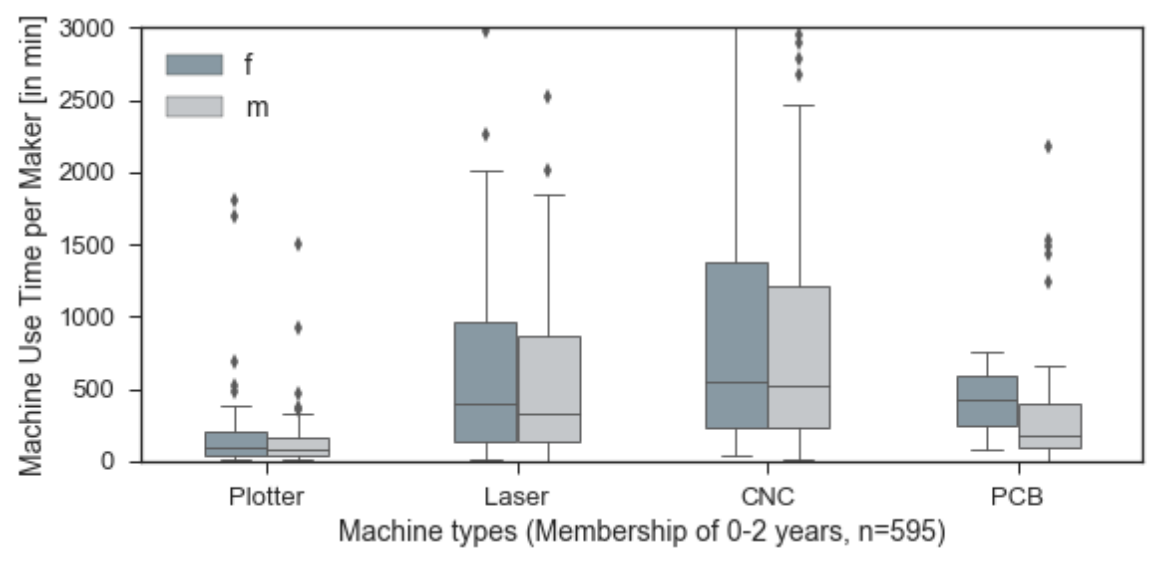

Fig. 11. Comparison of median use times based on machine type and gender 
Further we also tested for the statistical significance of the observed differences, i.e. what are the chances that we see differences where there are none. Again, applying the non-parametric Mann-Whitney $U$ test, all machines showed p-values substantially larger than .05 , hence no significant differences between genders can be claimed.

\section{Conclusion: Gender Gaps and Lack of Diversity are not Specific to FabLab Activities}

Summarizing the findings from the interviews, we have got statements indicating the importance of a respectful and supportive culture, the unwarranted genderisation of tasks and interests and the need for more female role models. The second part of the research was grounded in the logged activities of FabLab members and their demographic as well as membership data. The most overlap between the two data sets (interview transcripts and log data) was found in the area of analysing the actual behaviour of makers, at least their interactions with the machines.

Concerning a possible gender gap in FabLabs, the interviews revealed a strong link to early education and the wider presentation of women in tech. These are phenomena that originate largely outside the FabLab facilities, in families, kindergartens and schools. To a degree, we can see the same phenomenon reflected in the log data. The single most dominating difference are not varying use times but differences in absolute numbers between women and men joining a FabLab. There are four times more male makers than female makers in the first place, a gap that propagates into comparisons for specific machines, getting as high as 10 times more male makers than female makers trying printed circuit board etching. Other than that, both groups are relatively similar in age, female makers have slightly shorter memberships than male makers and are less prone to use 3d-printing or CNC milling machines. But then the two groups are comparable in their proportional preferences for the different membership types and the median use times spent with cutting plotters, CNC milling machines and laser cutters.

We are aware that a single FabLab is not representative for the whole maker movement, yet more than 3,000 makers and log data for about 4.5 years is a good starting point. Other FabLabs, such as the 'WeMake' FabLab in Milan, which is managed by a female maker, have gender ratios of $40 \%$ female makers versus $60 \%$ male makers (personal communication). However, the objective of studying gender relations or gender gaps within the maker movement cannot merely be to have equal shares of both genders. Hence, the debate should not get hijacked by surface numbers and more in-depth explorations are required, to better understand how to design spaces (physically and culturally) that are more attractive to a diverse range of people. 


\section{Outlook: Maker Spaces as Collective Awareness Platforms}

Collective awareness platforms have been described by three trends: social networking, pervasive computing (IoT, mobile platforms) and knowledge co-creation [23]. To some degree these trends are already part of people's everyday life, be it because they connect through Facebook, use a smartphone including its diverse tracking sensors or consult Wikipedia. In principle, these trends are value free, meaning that 1.86 billion monthly active Facebook users use the platform to promote social good as well as hate postings against dissenting groups [24].

Claiming value neutrality for 'maker spaces' is not an option. Society's deeply engrained cultural stereotypes and value preferences when relating gender with technology, engineering or making do not stop at the doors of a maker space. As we have shown, prejudices do in parts persist and female makers have concerns about how they are perceived, accepted and whether they are granted the same opportunities as their male counterparts.

What we can see is a huge potential for technological action - without implying technology determinism - where communities of any gender, race or age can be empowered. That this won't happen automatically has been shown in various studies portraying the typical maker as white, male and wealthy - including extreme cases with non-white memberships of $0 \%$ and female members between $10-30 \%$ [25].

As outlined above, discussing gender requires more in depths reflection about gendered life-worlds (e.g. childcare obligations, less economic flexibility for part-time workers or simply interests, that do not match 'smart home applications'). FabLabs as collective awareness platforms do not necessarily need to offer childcare or estitching workshops [13], in order to attract female makers. As highlighted in section four of this paper, change starts with appropriate language, communicating equal expectations for girls and boys, while accepting different approaches to making (regardless of gender). At the end, collective awareness platforms stand for potential activities as in 'makers can ...'. Aiming for more diverse maker spaces, we should avoid arguments including 'makers ought ...' [cf. 26]. Moral exhortations will not get us the change we need, instead, what we need are physical and organisational designs of maker spaces that encourage the desired behaviours we want to see.

\section{Acknowledgement}

This project has received funding from the European Union's Horizon 2020 research and innovation programme under grant agreement 688241.

\section{References}

1. Carstensen, T., Walter-Herrmann, J., Büching, C.: Gendered Fab Labs. Fab Lab: Of machines, makers and inventors. 53-64 (2014).

2. Wajcman, J.: Feminism confronts technology. Polity, Cambridge. (1991). 
3. Gershenfeld, N.: How to make almost anything: The digital fabrication revolution. Foreign Aff. 91, 43 (2012).

4. Voigt, C., Montero, C.S., Menichinelli, M.: An empirically informed taxonomy for the Maker movement. In: International Conference on Internet Science (INSCI 2016). Springer, Florence, Italy (2016).

5. Faulkner, W.: The technology question in feminism: A view from feminist technology studies. In: Women's studies international forum. pp. 79-95. Elsevier (2001).

6. Berg, A.-J., Lie, M.: Feminism and constructivism: Do artifacts have gender? Science, Technology, \& Human Values. 20, 332-351 (1995).

7. Bishu, S.G., Alkadry, M.G.: A Systematic Review of the Gender Pay Gap and Factors That Predict It. Administration \& Society. (2016).

8. Blackaby, D., Booth, A.L., Frank, J.: Outside Offers And The Gender Pay Gap: Empirical Evidence From the UK Academic Labour Market. The Economic Journal. 115, F81-F107 (2005).

9. Mills, M., Martino, W., Lingard, B.: Attracting, recruiting and retaining male teachers: Policy issues in the male teacher debate. British Journal of Sociology of Education. 25, 355-369 (2004).

10. Meadus, R.J.: Men in nursing: Barriers to recruitment. In: Nursing Forum. pp. 5-12. Wiley Online Library (2000).

11. Tiwari, R., Fischer, L., Kalogerakis, K.: Frugal innovation in scholarly and social discourse: An assessment of trends and potential societal implications. (2016).

12. Ames, M.G., Bardzell, J., Bardzell, S., Lindtner, S., Mellis, D.A., Rosner, D.K.: Making cultures: empowerment, participation, and democracy-or not? In: Proceedings of the extended abstracts of the 32nd annual ACM conference on Human factors in computing systems. pp. 1087-1092. ACM (2014).

13. Henry, L.: The Rise of Feminist Hackerspaces and How to Make Your Own. Model View Culture. 2, (2014).

14. Millard, J., Deljanin, S.R., Sorivelle, M.N., Munk, K.B., Langley, D., Broek, T. van den, Blankers, I., Voigt, C., Kieslinger, B., Unterfrauner, E., Pelka, B., Zirngieb, M.: D2.1 Conceptual and methodological framework, http://make-it.io/download/119/, (2016).

15. Mayring, P.: Qualitative Content Analysis. Forum: Qualitative Social Research [On-line Journal]. 1, (2000).

16. Unterfrauner, E., Voigt, C.: Makers' ambitions to do socially valuable things. Presented at the European Academy of Design Conference - EAD17, Rome, Italy (2017).

17. Palinkas, L.A., Horwitz, S.M., Green, C.A., Wisdom, J.P., Duan, N., Hoagwood, K.: Purposeful sampling for qualitative data collection and analysis in mixed method implementation research. Administration and Policy in Mental Health and Mental Health Services Research. 42, 533-544 (2015).

18. Turkle, S., Papert, S.: Epistemological pluralism: Styles and voices within the computer culture. Signs: Journal of women in culture and society. 16, 128-157 (1990).

19. Rasmussen, B., Håpnes, T.: Excluding women from the technologies of the future?: A case study of the culture of computer science. Futures. 23, 1107-1119 (1991).

20. Wuschitz, S.: Female Makers, http://www.p-art-icipate.net/cms/female-makers/, (2013).

21. Dunbar, R.: How many" friends" can you really have? IEEE Spectrum. 48, (2011). 
22. Waskom, M.: Seaborn Distribution Plot (Python Package), http://seaborn.pydata.org/tutorial/distributions.html, (2017).

23. Sestini, F.: Collective awareness platforms: Engines for sustainability and ethics. Technology and Society Magazine, IEEE. 31, 54-62 (2012).

24. Zephoria: The Top 20 Valuable Facebook Statistics - Updated May 2017, https://zephoria.com/top-15-valuable-facebook-statistics/, (2017).

25. Nascimento, S., Pólvora, A.: Maker Cultures and the Prospects for Technological Action. Science and Engineering Ethics. 1-20 (2016).

26. Ozbekhan, H.: The Triumph of Technology:" can" Implies" ought". System Development Corporation (1967). 